\title{
A PRÁTICA DA AUTOMEDICAÇÃO EM CRIANÇA POR PAIS E RESPONSÁVEIS
}

\author{
M.F.P. LIMA', D.B.S. LIMA' ${ }^{2}$, F.B.OLIVEIRA ${ }^{3}$, C.C.OLIVEIRA ${ }^{4}$, M.A. MACÊDO ${ }^{5}$, T.K.A.PEREIRA ${ }^{6}$
}

Estratégia Saúde da Família ${ }^{1}$, Universidade Estadual do Ceará ${ }^{2}$, Universidade Federal de Campina Grande ${ }^{3}$,

Universidade Regional do Cariri ${ }^{4}$, Núcleo de Apoio da Saúde da Família/Ceará ${ }^{5}$

ayrllesmacedo@hotmail.com ${ }^{5}$

Artigo submetido em 27/09/2016 e aceito em 23/06/2019

DOI: $10.15628 /$ holos.2019.5120

\section{RESUMO}

Objetivo: Analisar a prática da automedicação em crianças menores de dois anos por pais ou responsáveis na atenção primária. Métodos: Trata-se de um estudo descritivo com abordagem quantitativa. Participaram desta pesquisa 43 pais ou responsáveis que responderam a um questionário estruturado, no período de janeiro a fevereiro de 2016. Resultados: Os resultados mostraram que as mães já praticaram a automedicação em crianças menores de dois anos. A média da prática da automedicação foi de 2,7 vezes, sendo a utilização de medicamentos sem prescrição a prática mais frequente, motivados pelo conhecimento próprio e orientação do profissional de saúde. A febre, dor e gripe foram as situações clínicas motivadoras dos pesquisados para realizarem a automedicação. Conclusão: Torna-se fundamental a inclusão de programas de educação nas unidades de saúde, orientando os cuidadores sobre os riscos da prática da automedicação em crianças.

PALAVRAS-CHAVE: Automedicação, Crianças, Pais.

\section{PRACTICE OF SELF-MEDICATION IN CHILD BY PARENTS AND GUARDIAN}

\begin{abstract}
Objective: Analyze the practice of self-medication in children under two years of age by parents or guardians in primary care. Methods: This is a descriptive study with quantitative approach. This study included 43 parents or guardians who replied to a structured questionnaire, in the period from January to February of 2016. Results: The results showed that mothers have already practiced selfmedication in children under two years of age.
\end{abstract}

The average of the practice of self-medication was 2.7 times, and the use of non-prescription medicines being the most Common practice, motivated by self-knowledge and guidance of health professional. Fever, pain, and flue were the motivating clinical situation of those surveyed to carry out self-medication. Conclusion: It becomes crucial the inclusion of education programs in healthcare units, guiding caregivers about the risks of selfmedication in children.

KEYWORDS: Self-medication, Children, Parents. 


\section{INTRODUÇÃO}

No Estatuto da Criança e do Adolescente, considera-se criança, para os efeitos da Lei N.ㅇ 8.069 de 13 de julho de 1990, a pessoa até doze anos de idade incompletos (BRASIL, 2008). Nessa fase, a criança encontra-se na dependência direta de cuidados essenciais proporcionados pela mãe, em sua maioria, ou por um responsável, que vão desde higiene e nutrição até a administração de medicações no processo de saúde-doença.

Nesse contexto, alguns programas e políticas foram criados com o objetivo de fortalecer os cuidados com as crianças, como a ampliação do acesso aos serviços de saúde e a assistência de puericultura. Este se efetiva pelo acompanhamento periódico e sistemático das crianças para avaliação de seu crescimento e desenvolvimento, vacinação, orientações aos pais ou cuidadores sobre o aleitamento materno, alimentação no período do desmame e higiene individual. Além disso, é importante a identificação precoce dos agravos, através de visitas domiciliares no puerpério, consultas médicas, odontológica ou de enfermagem. Para isso, demanda a atuação de toda a equipe de saúde e multiprofissional que assiste a criança e sua família (BRASIL, 2012).

Dentre os cuidados com as crianças, Sousa et al (2013) relata que uma das partes integrantes do autocuidado é a prática da automedicação. Além disso, o simbolismo de saúde fortalece os hábitos do consumo, principalmente de medicamentos, de forma sedutora e vendável, como "alívio imediato da dor" dentre outros (URBANO et al, 2010).

Para a Organização Mundial da Saúde (OMS), a automedicação consiste na seleção e utilização de medicamentos isentos de prescrição para tratar doenças autolimitadas ou seus sintomas, estando inclusa no autocuidado (BECKHAUSER et al, 2010).

Por ser uma prática comum e variável de acordo com as sociedades, já é considerado um problema de saúde pública, pois fatores econômicos, políticos e culturais têm contribuído para o crescimento e a difusão da automedicação no Brasil e no mundo (LUCENA, 2007).

Beckhauser et al (2010) considera que a compra de medicamentos sem receita, o compartilhamento dos medicamentos com familiares ou amigos/vizinhos, a reutilização de sobras de medicamentos e a utilização de prescrições antigas são práticas de automedicação, assim como o descumprimento da prescrição, prolongando ou interrompendo o tratamento indicado. A prática da automedicação acompanhada da venda livre de medicamentos contribui, muitas vezes, para um consumo indevido e desnecessário. Para Urbano et al (2010) essa prática torna-se alvo de erros e acertos.

A escolha pela automedicação pode ser considerada favorável quando complementa o sistema de saúde, ajudando a descongestionar os atendimentos médicos e de enfermagem nas Unidades Básicas de Saúde (UBS), levando ao alívio de pequenas indisposições do paciente. Porém, quando imprópria, pode trazer potenciais riscos à saúde, como alergias, intoxicações e mascaramento da doença (MAGALHÃES, 2014), bem como prolongar o tempo para a procura do serviço de saúde em situações de maior gravidade, particularmente, quando se trata de crianças (SOUSA et al, 2013). 
Mesmo sendo parte integrante do autocuidado, a automedicação deve ser realizada de forma racional. Neste sentido, as crianças representam um grupo susceptível ao uso irracional, principalmente em função da grande disponibilidade de medicamentos e reduzido número de estudos no uso e desenvolvimento destes envolvendo as crianças, sendo que com frequência as doses utilizadas são ajustadas em relação às doses testadas em adultos (SOUSA et al, 2013).

Segundo Ascari et al (2014), a Secretaria de Políticas de Saúde do Ministério da Saúde, com o propósito de garantir segurança, eficácia e qualidade dos medicamentos, o uso racional e o acesso da população a medicação essencial, aprovou a Política Nacional de Medicamentos conforme Portaria no 3.916 de 30 de outubro de 1998. A implantação dessa política fortalece as diretrizes e princípios legalmente estabelecidos, explicando quais são as responsabilidades dos gestores dentro do Sistema Único de Saúde (SUS), nas três esferas de governo, devendo atuar em parceria para a implantação e efetivação da política e assegurar o acesso da população a medicamentos seguros, eficazes e de qualidade, ao menor custo.

A promoção do uso racional de medicamentos é um dos objetivos a serem alcançados pela Política Nacional de Medicamentos com ênfase ao processo educativo para os usuários, consumidores, profissionais e na formação profissional abordando os riscos da automedicação, da interrupção e da troca da medicação prescrita (ASCARI et al, 2014).

O interesse por esse tema surgiu a partir de relatos de mães ou responsáveis sobre a administração de algumas medicações por iniciativa própria ou por indicações de amigos, familiares, em sua maioria as avós e por balconistas de farmácias, nos atendimentos de enfermagem às crianças na UBS. Essa ação foi realizada com o intuito de aliviar sinais e sintomas de alguma doença apresentados pela criança e percebidos pelos pais e/ou responsáveis antes de procurarem atendimento de um profissional habilitado.

Nesse contexto, torna-se evidente a importância de estudos que identifiquem comportamentos relacionados à automedicação em crianças por pais ou responsáveis. Assim sendo, espera-se que os resultados desse estudo sejam utilizados para instigar a reflexão e sensibilizar a população, principalmente os cuidadores de crianças, sobre o uso racional dos medicamentos.

Enfim, o objetivo geral deste estudo foi analisar a prática da automedicação em crianças menores de dois anos por pais ou responsáveis, tendo como objetivos específicos: caracterizar o perfil sociodemográfico dos pais ou responsáveis das crianças menores de dois anos cadastradas e acompanhadas pela Equipe de Referência em Saúde da Família; verificar as situações clínicas que levam à prática da automedicação; identificar os fármacos mais utilizados na prática da automedicação e descrever os eventos adversos apresentados pelas crianças automedicadas.

\section{METODOLOGIA}

Trata-se de um estudo descritivo com abordagem quantitativa. A pesquisa é considerada descritiva, por ter como objetivo primordial a descrição de características de determinada população e uma de suas características mais significativas está na utilização de técnicas 
padronizadas de coletas de dados como o questionário (GIL, 2006). Quantitativo por traduzir em números, as opiniões e informações para classificá-las e analisá-las (KAUARK, 2010).

O estudo foi realizado na UBS Maria Rita Pereira dos Santos, localizada no Bairro Vila Centenário no município de Iguatu, Ceará, Brasil. A escolha da referida unidade deu-se, principalmente, por ser um dos cenários de prática da Residência Integrada em Saúde da Escola de Saúde Pública do Ceará, no respectivo município.

A UBS oferece atendimento ambulatorial de segunda à sexta-feira, no período da manhã e tarde. Sua área de abrangência tem, aproximadamente, 975 famílias cadastradas e acompanhadas, contemplando, com seus serviços, cerca de 3.132 habitantes. Possui uma equipe completa da Estratégia Saúde da Família (ESF), composta por uma médica intercambista, uma enfermeira, uma técnica de enfermagem, sete agentes comunitárias de saúde (ACS), uma dentista, uma auxiliar de saúde bucal e uma enfermeira residente. A UBS é apoiada pelo Núcleo de Apoio à Saúde da Família (NASF) residente, composta por uma assistente social, uma psicóloga, uma nutricionista e um fisioterapeuta.

Segundo os dados do Sistema de Informação da Atenção Básica (SIAB), no mês de janeiro de 2016, havia 57 crianças menores de dois anos cadastradas e acompanhadas pela equipe da ESF.

Para a obtenção da amostra foi utilizada a fórmula para cálculo de amostra finita e adotouse a prevalência de automedicação estimada em $56 \%$, conforme estudo sobre automedicação em crianças e adolescentes (PEREIRA et al, 2007), intervalo de confiança de 95\% e erro de estimação de $4 \%$, resultando em uma amostra de 52 crianças.

$$
n=\frac{N \cdot \hat{p} \cdot \hat{q} \cdot\left(Z_{\alpha / 2}\right)^{2}}{\hat{p} \cdot \hat{q} \cdot\left(Z_{\alpha / 2}\right)^{2}+(N-1) \cdot E^{2}}
$$

Onde:

$\mathrm{n}=$ Tamanho da amostra.

$\mathrm{N}=$ Tamanho da população.

$Z_{\alpha / 2}=$ Valor crítico que corresponde ao grau de confiança desejado. Considerou-se $\alpha=$ $5 \%$, logo o valor positivo de z para a área acumulada sob a curva normal padronizada $(0,97500)$ é 1,96 .

$p=$ Percentagem com a qual o fenômeno se verifica.

$q=$ Percentagem complementar (100-p).

$E^{2}=$ Erro máximo permitido.

A coleta de dados foi realizada no mês de janeiro de 2016, por meio de um formulário estruturado aplicado aos pais ou responsáveis, nas dependências da UBS, enquanto eles 
aguardavam a consulta médica ou de enfermagem das crianças. Posteriormente, em fevereiro de 2016, foram realizadas visitas domiciliares àqueles que não comparecerem na UBS durante o período da coleta de dados.

O formulário foi aplicado aos pais ou responsáveis devido a inviabilidade de ser aplicado em crianças menores de dois anos. Como critério de inclusão no estudo foi adotado a prática da automedicação realizada pelos pais ou responsáveis nas crianças menores de dois anos, pelo menos uma vez. Foram excluídas nove crianças, pois os pais ou responsáveis não foram encontrados nos domicílios, resultando em uma amostra de 43 crianças.

A análise se deu por tabulação dos dados seguida de cálculos estatísticos descritivos, utilizando software Statistical Package for the Social Sciences (SPSS) versão 20.0 e foram apresentados por tabelas com respectiva interpretação das mesmas.

De acordo com os aspectos éticos da Resolução do Conselho Nacional de Saúde, № 466 de 12 de dezembro de 2012 (BRASIL, 2012), os sujeitos que aceitaram participar da pesquisa foram informados sobre a natureza, objetivos e benefícios da pesquisa com a leitura detalhada e assinatura do Termo de Consentimento Livre e Esclarecido, ficando-Ihes assegurado o direito à privacidade. Foram, ainda, garantidos os direitos de confidencialidade, anonimato, proteção da imagem e não estigmatização, além da não utilização das informações em prejuízo das pessoas, inclusive em termo de autoestima, de prestígio e/ou econômico-financeiro.

O projeto foi encaminhado para apreciação (CAAE: 5196975.0000.5037) do Comitê de Ética da Escola de Saúde Pública do Estado do Ceará (ESP/CE) no dia 17/12/2015, onde foi aprovada a viabilidade de sua realização, com o parecer $n^{\circ}: 1374716$.

\section{RESULTADOS}

Dos 43 participantes elegíveis, $14 \%$ dos pais ou responsáveis haviam praticado a automedicação em crianças menores de dois anos pelo menos uma vez, e cerca de um terço o fizeram por duas e três vezes, correspondendo a 34,9\% e 32,6\%, respectivamente. Dos pesquisados, $100 \%$ são do sexo feminino, as cuidadoras foram representadas, em sua maioria, pelas próprias mães (83,7\%). A média de idade entre elas foi de 31,7 anos (DP= $=13,68)$, variando entre 15 e 72 anos de idade.

Na tabela 1 pode-se visualizar que a raça predominante foi parda $(67,4 \%)$ e religião católica (72,1\%). Em relação à escolaridade, prevaleceu o ensino médio completo (48,8\%), seguido do ensino fundamental incompleto (25,6\%). Metade das entrevistadas referiu união estável $(51,2 \%)$ como situação conjugal atual. As ocupações mais comuns foram os serviços não remunerados $(69,8 \%)$, mencionando as tarefas domésticas como ocupação diária. A renda familiar dominante entre as pesquisadas foi menor ou igual a um salário mínimo $(65,1 \%)$. Em relação à situação de moradia, a frequência encontrada entre as famílias que moram em casas próprias foi igual àquelas que moram em casas alugadas (44,2\%). Um pouco mais da metade das famílias são beneficiárias do Programa Bolsa Família $(53,5 \%)$. 
TABELA 1: Características sócio demográficas dos pais ou responsáveis que praticam automedicação em crianças. Iguatu-Ceará- Brasil, 2016.

\begin{tabular}{|c|c|c|}
\hline VARIÁVEIS & $\mathbf{N}$ & $\%$ \\
\hline \multicolumn{3}{|l|}{ Raça } \\
\hline Parda & 29 & 67,4 \\
\hline Branca & 10 & 23,3 \\
\hline Negra & 04 & 9,3 \\
\hline \multicolumn{3}{|l|}{ Religião } \\
\hline Católica & 31 & 72,1 \\
\hline Evangélica & 08 & 18,6 \\
\hline Outras & 04 & 9,3 \\
\hline \multicolumn{3}{|l|}{ Escolaridade } \\
\hline Ensino médio completo & 21 & 48,8 \\
\hline Ensino Fundamental Incompleto & 11 & 25,6 \\
\hline Ensino Médio Incompleto & 07 & 16,3 \\
\hline Analfabeto & 04 & 9,3 \\
\hline \multicolumn{3}{|l|}{ Estado Civil } \\
\hline União estável & 22 & 51,2 \\
\hline Casada & 12 & 27,9 \\
\hline Solteira & 05 & 11,6 \\
\hline Viúva & 03 & 7,0 \\
\hline Separada & 01 & 2,3 \\
\hline \multicolumn{3}{|l|}{ Ocupação } \\
\hline Trabalho não remunerado (dona de casa) & 30 & 69,8 \\
\hline Trabalho remunerado & 13 & 30,2 \\
\hline \multicolumn{3}{|l|}{ Renda Familiar } \\
\hline$<1 \mathrm{SM}^{*}$ & 28 & 65,1 \\
\hline DE 1 A 2 SM & 11 & 25,6 \\
\hline$>2 \mathrm{SM}$ & 04 & 9,3 \\
\hline \multicolumn{3}{|l|}{ Situação de Moradia } \\
\hline Própria & 19 & 44,2 \\
\hline Alugada & 19 & 44,2 \\
\hline Cedida & 05 & 11,6 \\
\hline
\end{tabular}




\begin{tabular}{ccc}
\hline Beneficiário do Bolsa Família & & \\
\hline Sim & 23 & 53,5 \\
Não & 20 & 43,5 \\
\hline
\end{tabular}

*Salário mínimo vigente $(\mathrm{R} \$ 880,00)$

As crianças automedicadas foram representadas, em sua maioria, pelo sexo masculino $(53,5 \%)$ e faixa etária, os menores de um ano (60,5\%). A raça dominante foi parda $(60,5 \%)$. Somente 9,3\% das crianças possuíam plano de saúde, sendo a maioria dependente dos serviços públicos de saúde (Tabela 2).

TABELA 2: Características sociodemográficas das crianças automedicadas por pais ou responsáveis. Iguatu-Ceará-Brasil, 2016.

\begin{tabular}{lcc}
\hline \multicolumn{1}{c}{ VARIÁVEIS } & N & \% \\
\hline Sexo & & \\
\hline Masculino & 23 & 53,5 \\
Feminino & 20 & 46,5 \\
\hline Faixa Etária & & 60,5 \\
\hline$<1$ ano & 26 & 39,5 \\
1 a 2 anos & 17 & \\
\hline Raça/Cor & & 60,5 \\
\hline Parda & 26 & 39,5 \\
Branca & 17 & \\
\hline Plano de Saúde & & 90,7 \\
\hline Não & 39 & 9,3 \\
\hline Sim & 04 & \\
\hline
\end{tabular}

Os dados relacionados à prática da automedicação estão apresentados na Tabela 3, onde a média encontrada da quantidade de vezes que as pesquisadas praticaram a automedicação foi de

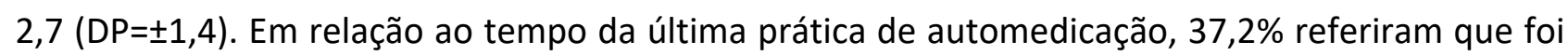
em até 7 dias antes da data em que foram entrevistadas. Quando investigadas sobre as formas de automedicação, a mais comum foi utilização de medicamentos sem receita de um profissional prescritor (93\%), seguida por reutilização de sobras de medicamentos $(65,1 \%)$, reutilização de prescrições anteriores $(30,2 \%)$ e alteração da prescrição médica $(16,3 \%)$. Os fatores que mais influenciaram a prática da automedicação foram conhecimento próprio $(72,1 \%)$ e orientação do profissional de saúde $(69,8 \%)$. 
TABELA 3: Formas de automedicação praticadas em crianças por pais ou responsáveis. Iguatu-CearáBrasil, 2016.

\begin{tabular}{|c|c|c|}
\hline VARIÁVEIS & n & $\%$ \\
\hline \multicolumn{3}{|l|}{ Última prática da automedicação } \\
\hline Até 7 dias & 16 & 37,2 \\
\hline$>30$ dias & 13 & 30,2 \\
\hline 8 a 15 dias & 06 & 14,0 \\
\hline 16 a 30 dias & 08 & 18,6 \\
\hline Utilização de medicamentos sem receita & 40 & 93,0 \\
\hline Reutilização de sobras de medicamentos & 28 & 65,1 \\
\hline Reutilização de prescrições anteriores & 13 & 30,2 \\
\hline Alteração de prescrição médica & 07 & 16,3 \\
\hline \multicolumn{3}{|c|}{ Fatores que influenciaram a prática da automedicação } \\
\hline Conhecimento próprio & 31 & 72,1 \\
\hline Orientação do profissional de saúde & 30 & 69,8 \\
\hline Orientação adquirida na farmácia & 26 & 60,5 \\
\hline Familiares & 18 & 41,9 \\
\hline Vizinhos & 13 & 30,2 \\
\hline Amigos & 12 & 27,9 \\
\hline Propaganda & 01 & 2,3 \\
\hline
\end{tabular}

De acordo com a Tabela 4, a febre foi a situação clínica mais referida como motivo para a prática da automedicação (93\%), 44,2\% citaram dor e 37,2\% gripe. Vale ressaltar que 53,5\% mencionaram até duas situações clínicas apresentadas pela criança. Dos medicamentos, os antitérmicos foram os mais utilizados $(88,4 \%)$, seguidos por analgésicos $(53,5 \%)$, polivitamínicos $(30,2 \%)$ e soro nasal $(27,9 \%)$, sendo que a maioria $(62,8 \%)$ possui o hábito de ler a bula das medicações.

Evidenciou-se que há reserva de medicamento nas residências de todas as entrevistadas para uma eventualidade, sendo que a maioria estoca medicamentos em depósitos com tampa, posicionados em cima do armário da cozinha. Quando questionados sobre a ocorrência de efeitos indesejados nas crianças após a automedicação, apenas 7\% afirmaram ter percebido tais efeitos. Destas, $100 \%$ apresentaram exantema no corpo e seus cuidadores suspenderam a medicação. 
TABELA 4: Situações clínicas e medicamentos utilizados na prática da automedicação em crianças por pais ou responsáveis. Iguatu-Ceará-Brasil, 2016.

\begin{tabular}{|c|c|c|}
\hline VARIÁVEIS & $\mathbf{n}$ & $\%$ \\
\hline \multicolumn{3}{|c|}{ Situações clínicas que levam à prática da automedicação } \\
\hline Febre & 40 & 93,0 \\
\hline Dor & 19 & 44,2 \\
\hline Gripe & 16 & 37,2 \\
\hline Coriza nasal & 12 & 27,3 \\
\hline Diminuição de Apetite & 11 & 25,6 \\
\hline Cólica & 10 & 26,3 \\
\hline Diarreia & 09 & 20,9 \\
\hline Tosse & 01 & 2,3 \\
\hline Vômito & 01 & 2,3 \\
\hline Constipação & 01 & 2,3 \\
\hline \multicolumn{3}{|l|}{ Medicamentos utilizados } \\
\hline Antitérmicos & 38 & 88,4 \\
\hline Analgésicos & 23 & 53,5 \\
\hline Polivitamínicos & 13 & 30,2 \\
\hline Soro nasal & 12 & 27,9 \\
\hline Xarope expectorante & 11 & 25,6 \\
\hline SRO & 04 & 9,3 \\
\hline Antibióticos & 03 & 7,0 \\
\hline Antiespasmódicos & 03 & 7,0 \\
\hline Vitamina $\mathrm{C}$ & 03 & 7,0 \\
\hline Antiinflamatórios & 02 & 4,7 \\
\hline Antialérgicos & 01 & 2,3 \\
\hline Laxante & 01 & 2,3 \\
\hline
\end{tabular}

SRO: Sais de reidratação oral.

\section{DISCUSSÃO}

No presente estudo, todos os pesquisados são do sexo feminino, representadas, em sua maioria, pelas mães das crianças menores de dois anos. Beckhauser et al (2010), em um estudo transversal com os responsáveis pelas crianças com idade entre zero e 14 anos, em seus respectivos domicílios, encontraram que $95 \%$ dos responsáveis e/ou cuidadores de crianças compõem o grupo 
de mães. Urbano et al (2010), em sua pesquisa, enfatizam que as mães representam $65 \%$ dos responsáveis pela automedicação em crianças. Isso demonstra que a participação materna nos cuidados diários de seus filhos ainda está fortalecida, como também a prática da automedicação infantil, pelas mães, é incisiva.

Com relação ao grau de escolaridade das entrevistadas e renda das famílias das crianças participantes da pesquisa, constatou-se que quase a metade concluiu o ensino médio e $65,1 \%$ vivem com até um salário mínimo. Renda provida, em maioria, pelos companheiros, já que 69,8\% são cuidadoras do lar. Em estudo realizado por Moraes et al (2013), cujo objetivo foi investigar a utilização de medicamentos por pais ou cuidadores de crianças entre zero e seis anos de idade residentes em domicílios da área urbana de Bagé, mostrou que $59 \%$ das famílias vivem com mais de um salário mínimo e 53,3\% possui, no mínimo, o ensino fundamental completo como grau de escolaridade. O nível de escolaridade elevado parece estar mais relacionado com a prática da automedicação em crianças, talvez, pelo motivo de os responsáveis possuírem uma maior compreensão das prescrições e bulas, dando maior segurança aos pais ou responsáveis para realizarem tal prática.

A prevalência da prática da automedicação por até três vezes (81,5\%), em crianças menores de dois anos foi maior que estudos realizados anteriormente. Oliveira et al (2010) mostram a prevalência do uso de medicamentos em crianças de três a doze meses de idade de aproximadamente 65\% e, aos 24 meses, de 55\%. Urbano et al (2010), ao avaliarem o uso de medicamentos em crianças de 0 a 15 anos de idade, sem a devida prescrição médica, em diferentes pontos das cidades de Santos e São Vicente, encontraram prevalência de $62 \%$. A pesquisa que mais se aproximou da atual foi a de Beckhauser et al (2010), onde referiram que a automedicação ocorreu em $77,5 \%$ dos entrevistados.

Para Telles e Pereira (2013), a automedicação em crianças menores de cinco anos merece destaque, pois há maior probabilidade de reutilizarem antigas prescrições em relação às crianças mais velhas, ou seja, a automedicação ocorre em maior prevalência nessa faixa etária.

Pouco mais da metade dos pais ou responsáveis das crianças incluídas no estudo utilizou algum medicamento nos 15 dias que antecederam a entrevista. A prevalência estimada é semelhante ao estudo de Moraes et al (2014) que, no mesmo período, encontraram prevalência de $52 \%$. É possível que isso ocorra em decorrência da dificuldade de acesso aos serviços públicos de saúde, que em situações de urgência/emergência, levam às pessoas a buscarem outros tipos de acesso às medicações para o alívio de suas enfermidades e de seus familiares.

A aquisição de medicamento de forma gratuita pelo Sistema Único de Saúde (SUS) apresenta um fator de proteção para a automedicação, uma vez que para ter acesso faz-se necessário realizar consulta médica e obter a prescrição do medicamento (ASCARI et al, 2014).

A utilização de medicamentos sem prescrição médica foi a forma de automedicação mais referida entre as entrevistadas, onde os fatores que mais as influenciaram foram o conhecimento próprio, seguidos pela orientação do profissional de saúde e orientações adquiridas nas farmácias. Para Beckhauser et al (2010), a automedicação é praticada, principalmente, pela utilização de 
medicamentos sem receita de um profissional prescritor, seguida por reutilização de prescrições antigas ou sobras de medicamentos.

A heterogeneidade dos métodos utilizados em outros estudos dificulta a comparação dos dados, visto que o período recordatório e, principalmente, a faixa etária investigada têm grande variação nos diferentes trabalhos realizados. Outra diferença encontrada foi a origem do uso dos medicamentos, enquanto alguns estudos investigaram o uso de medicamentos por indicação médica outros avaliaram o uso por automedicação (MORAES et al, 2014).

Dentre as situações clínicas que, espontaneamente, foram elencadas pelas pesquisadas, a febre foi a mais mencionada, seguida por dor e gripe. Outro estudo mostrou a relação das doenças que as mães entrevistadas justificaram a automedicação, corroborando com os resultados encontrados: febre (57,6\%), dor (27,2\%) e gripe (4,3\%) (BECKHAUSER et al, 2010). Já para Medeiros, Pereira e Medeiros (2011), a febre (50\%), gripe (25\%), resfriado (13\%) e infecção na garganta (12\%) foram as situações mais relatadas. As condições clínicas referidas parecem ser de fácil manejo, pois os medicamentos utilizados para o alívio são bastante conhecidos, facilmente adquiridos e comercializados em farmácias, sem prescrição médica. Fato que contribui para o uso irracional de determinados medicamentos.

Os grupos de medicamentos mais utilizados foram os analgésicos e antitérmicos, com preferência para o paracetamol e dipirona, podendo ser justificado pela elevada prevalência da febre entre as crianças menores de dois anos. Este sintoma é preocupante, uma vez que serve como sinal de alerta para os pais de que algo de errado está acontecendo, e estima-se que aproximadamente $25 \%$ de todas as consultas na emergência pediátrica se devem à febre (TROTTA; GILIO, 1999).

No estudo de Perdizes et al (2015), os analgésicos/antitérmicos foram o segundo grupo farmacológico mais utilizado (18,7\%), prescritos ou não, em crianças que frequentavam uma creche municipal na cidade de Santos. Beckhauser et al (2010), relataram em seu estudo que as principais classes medicamentosas utilizadas na automedicação foram os analgésicos e antitérmicos (75\%), representados pelo paracetamol (45\%) e dipirona (15\%).

Para Moraes et al (2013), o paracetamol e o ibuprofeno estão na lista de medicamentos para infância da Organização Mundial de Saúde e a dipirona, analgésico/antitérmico de baixo custo, é integrante da lista de medicamentos do Programa Farmácia Popular.

As crianças automedicadas, em sua maioria, não apresentaram reação alérgica após serem automedicadas (93\%), dados que corroboram com o estudo de Romão, Toledo e Soares (2014), onde $95 \%$ afirmaram não ter percebido a ocorrência de efeitos indesejados dos medicamentos nas crianças.

Nesse ínterim, percebe-se que a prática da automedicação está inserida na dinâmica do cotidiano social e familiar, os medicamentos são cada vez mais procurados e consumidos para o alívio imediato de sinais e sintomas apresentados, por mais limitados que sejam. Logo, é imperativo que os profissionais de saúde, principalmente os da ESF, que visitam diariamente as famílias, orientem os pais ou responsáveis de crianças, sejam em qualquer idade, sobre os riscos da automedicação e incentivem à procura dos serviços de saúde, quando necessário. 


\section{CONSIDERAÇÕES FINAIS}

Conclui-se que a automedicação em crianças menores de dois anos é relevantemente praticada no dia a dia e geralmente ocorre sob a responsabilidade das mães, em sua maioria, em situações de febre ou dor, seja por conhecimento próprio ou orientado por um profissional de saúde. As medicações mais utilizadas foram os analgésicos ou antitérmicos, com o intuito de aliviar o sofrimento físico dessas crianças em tempo hábil, antes mesmo de procurar um atendimento por um profissional de saúde.

Diante do contexto, existe uma necessidade vigente em diminuir essa prática, através da informação à população sobre o uso racional de medicamentos, principalmente na infância, considerando a qualidade, a segurança e a eficácia dos fármacos como também incentivar a procura e o acesso ao profissional de saúde.

\section{REFERÊNCIAS}

ASCARI, R. A. et al. (2014). Estratégia Saúde da Família: automedicação entre usuários. Chapecó, SC: Revista Uningá. Vol 18, n.2, pp.42-47.

BECKHAUSER, G. C. et al. (2010). Utilização de medicamentos na Pediatria: a prática de automedicação em crianças por seus responsáveis. Florianópoles, SC: Rev Paul Pediátrica. p.262-268.

BRASIL. Conselho Nacional de Saúde. Resolução n 466 de 12 de dezembro de 2012. Brasília, DF.

BRASIL. (2008). Estatuto da criança e do adolescente: Lei federal no 8069, de 13 de julho de 1990. 3. Brasília, DF.

BRASIL. (2012). Saúde da criança: crescimento e desenvolvimento. (Cadernos de Atenção Básica, noㅡ 33). Brasília, DF. 272 p.

GIL, A. C. (2006). Como Elaborar Projetos de Pesquisa. 4. ed. São Paulo, SP: Atlas.

KAUARK, F. S. et al. (2010). Metodologia da pesquisa: um guia prático. Itabuna, BA: Via Litterarum. 88p.

LUCENA, A. L. (2007). Análise da prática da automedicação, no município de Brejo Santo, nas áreas assistidas pela Estratégia Saúde da Família - ESF. Brejo Santo, CE, 2007. Monografia (Especialização em Assistência Farmacêutica) - Escola de Saúde Publica do Ceará.

MAGALHÃES, F. C. (2014). Práticas populares de cuidados à criança: o saber/fazer de cuidadoras. Natal-RN, 2014. Dissertação (Mestrado em Enfermagem) - Universidade Federal do Rio Grande do Norte.

MEDEIROS, R. A.; PEREIRA, V.G.; MEDEIROS, S.M. (2011). Vigilância em saúde na enfermagem: o caso das medicações sem prescrição em crianças. Natal, RN: Escola Anna Nery. p. 233-237.

MORAES, C. G. et al. (2013). Utilização de medicamentos entre crianças de zero a seis anos: um 
estudo de base populacional no sul do Brasil. Rio de Janeiro, RJ: Ciência \& Saúde Coletiva. 18(12).

OLIVEIRA, E. A. et al. (2010). Uso de medicamentos do nascimento aos dois anos: coorte de nascimentos de Pelotas, RS. Pelotas, RS: Rev Saúde Pública. p.591-600.

PEREIRA, F.S.V.T. (2007). Automedicação em crianças e adolescentes. Porto Alegre, RS: J. Pediatria. $8(5)$.

PERDIZES, G. O. et al. (2015, jul-set). Uso de medicamentos em crianças de creche na cidade de Santos. Santos, SP: Revista UNILUS Ensino e Pesquisa. 12(28).

ROMÃO, M. M. S.; TOLEDO, T. R.; SOARES, A.F. (2014). Uso de medicamentos por crianças de escolas públicas de um munícipio da Zona da Mata Mineira. Muriaé, MG: Revista Científica da Faminas. 10(2).

SOUSA, M. S. et al (2013). Automedicação em crianças que procuram o serviço de emergência em um hospital no sul do Brasil. Tubarão, SC: Rev. Bras. Farm. p.54-58.

TELLES, P. C. P. F.; PEREIRA, A. C. J. (2013). Automedicação em crianças de zero a cinco anos: fármacos administrados, conhecimentos, motivos e justificativas. Diamantina, MG: Esc Anna Nery (impr.). p.291-297.

TROTTA, E. A.; GILIO, A. E. (1999). Febre aguda sem sinais de localização em crianças menores de 36 meses de idade. Rio de Janeiro (RJ): Jornal de Pediatria. 75(2), p.214-222.

URBANO, A. Z. R. et al. (2010). Automedicação Infantil: o uso indiscriminado de medicamentos nas cidades de Santos e São Vicente. [Santos, SP]: Revista Ceciliana. 